\title{
Latinoamérica en la Comunicación Mundial
}

Dra. Ma Antonia Martín Díez

Universidad Europea de Madrid

Visión genérica de la comunicación en América Latina por medio de diferentes apartados en los que se reflejan desde la dependencia de los Estados Unidos hasta los logros e intentos de desarrollar medios de comunicación que busquen y profundicen en las raíces culturales latinoamericanas. El surgimiento de grandes grupos autóctonos de comunicación y sus alianzas con grupos de los países desarrollados es otro de los aspectos a resaltar.

a estructura de la comunicación latinoamericana se encuentra inmersa dentro del sistema internacional de la información ${ }^{1}$. En ella encontramos, obviamente, las tendencias de intercambio e interdependencia actuales. Estas se pueden producir bien como relaciones paralelas (junto a), bien como relaciones opuestas (frente a).

Entre las numerosas tendencias que subyacen en la estructura de la comunicación latinoamericana cuya investigación nos ha llevado a descubrir, destacamos las siguientes:

1-. Regionalización versus globalización.

2-. Poder autónomo versus poder dependiente.

3-. Proteccionismo versus librecambismo.

4-. Estatismo versus privatización.

5-. Civilización occidental versus otras civilizaciones

6-. Culturas propias versus cultura norteamericana.

7-. Exposición ideológica directa versus método del entretenimiento.

Ver sobre el tema, S. NÚÑEZ DE PRADO y Ma A. MARTÍN, Estructura de la comunicación mundial, Madrid, Univérsitas, pp. 61-78. 
Vamos a recorrer cada uno de esos grupos de tendencias:

\section{I)-. Regionalización versus globalización}

La comunicación internacional se ha desarrollado bajo el importante influjo de la economía y de la técnica. Es lo que ha condicionado -como dice Armand Mattelart-, no sólo las estrategias sino también las teorías. Pero la economía y la técnica, a partir de determinado momento, comenzaron a ser superadas por la cultura. «La primacía de la cultura -dice el autor mencionado-, no alcanzaría su legitimación hasta muy recientemente, en el transcurso de los años ochenta $»^{2}$.

El cambio tuvo diversas causas como la crisis del estatismo con la potenciación del factor localista, la valoración de las identidades y la emulación producida desde las diferencias y las desigualdades.

Lo que vamos diciendo aquí adquiere una dimensión más reivindicativa al plantearnos si el imperialismo cultural no es más que un mito. Abunda en ello un autor como Claude-Jean Bertand cuando dice que «la lengua, la historia y la cultura de un país secular tienen sobrada fuerza para mantener su identidad, incluso frente a un vecino muy cercano desde siglos atrás $»^{3}$. Claro que dicho autor plantea la excepción de la colonización muy prolongada. ¿Es éste el caso de Latinoamérica?.

La consagración del término globalización coincide con la liberalización y privatización de las redes de comunicación. Este proceso, se inició en los años setenta en los Estados Unidos con la liberalización de las actividades bancarias, pero se extendió realmente a partir del desmantelamiento en 1984 de la sociedad ATT (American Telegraph \& Telephone). Fue impulsada por la mayoría republicana con el objetivo de eliminar la normativa anti-trust en los sectores de televisión y telecomunicaciones. La reforma permite a las empresas telefónicas y de televisión competir en los respectivos sectores y a las compañías de larga distancia como AT \& T y MCI entrar en el mercado regional controlado por las siete Bell regionales y a éstas, a su vez, entrar en el mercado internacional. Así mismo, a partir del 31 de marzo de 1999, los operadores de cable ofrecen telefonía y las compañías telefónicas servicios de vídeo. Para completar este marco, una sentencia dictada por un juez federal de Los Angeles (noviembre de 1994) permite a las cadenas nacionales su integración con los estudios de cine. Esto ha hecho que las productoras como Fox, Warner o Paramount creen sus propias cadenas generalistas. En la anterior regulación las cadenas tradicionales no podían participar en los

2 A. MATTELART, La Comunicación mundo. Historia de las ideas y de las estrategias, Madrid, Fundesco, 1993, p.15

3 J.C. BERTRAND, "Grandes modelos de sistemas informativos del mundo", en J. BENGOECHEA, Situación 1995, Bilbao, 1994, p.25. 
beneficios que daban las películas y programas de las productoras. El cambio ya se había producido en Europa y en América Latina. Así en Europa, en el Consejo de Ministros de Telecomunicaciones celebrado en Bruselas el 17 de noviembre de 1994, se decidió la liberalización de las telecomunicaciones para las mismas fechas que la telefonía vocal (1 de enero de 1998). Desde el 1 de enero de 1996, a través de una directiva de la Comisión las redes de televisión por cable, pueden proporcionar todo tipo de servicios multimedia. En cambio, la red de telefonía ha tenido que esperar a 1998 para hacer lo propio, lo cual ha provocado quejas por parte de las compañías telefónicas. El retraso sin embargo, tiene su lógica si se piensa en los distintos grados de penetración del cable en los estados miembros ${ }^{4}$.

En Latinoamérica, la transición se inició en 1988 con las privatizaciones en Chile, luego extendidas a México, Argentina, Venezuela, Perú, Bolivia y Brasil, y el aumento de inversiones estatales en infraestructuras físicas. Debido, a esta tendencia universal y a factores propios como «las obligaciones económicas que lastraban unas finanzas públicas ahogadas por un alto nivel de endeudamiento, el importante aumento de la demanda, tanto en los segmentos tradicionales del mercado como en los nuevos servicios, así como la diversificación tecnológica en la oferta», se han ido quebrando las bases de los monopolios.

Esta tendencia logró dimensiones auténticamente universales tras el Acuerdo de la Organización Mundial del Comercio (OMC), el pasado 15 de febrero de 1997.

\section{II)-. Poder autónomo versus poder dependiente}

El poder dependiente es el ligado a las estructuras norteamericanas y europeas presentes en América Latina. Se formó con los repartos por zonas de influencia que realizaron las agencias informativas. Associated Press a partir del Acuerdo de París (5 de octubre de 1902) se expansionó por los territorios de Cuba, Puerto Rico, Hawai, Islas Filipinas y América Central. Su presencia en América Latina se consolidó durante la I Guerra Mundial, aprovechando la rivalidad francoalemana. Su influencia actual es menor debido a la expansión de la Agencia Efe y a los proyectos de cooperación entre las agencias de la zona. Sin embargo, a diferencia de Europa, no se fundaron agencias nacionales públicas, durante el periodo de entreguerras (con la excepción de Telam en Argentina o la Agencia Peruana de Noticias y Publicidad S. A.). Hoy día hay incluso Estados que dejan esta iniciativa a la Iglesia (Bolivia- Agencia de Noticias Fides) o a los grupos de comunicación (Brasil- Globo). La eficacia propagandística no es por ello menos valorada. Los movimientos guerrilleros centroamericanos han creado sus propias agencias (Siagpres en Guatemala, Notisal-Venceremos en El Salavador, Hondu-

4 Ver sobre el tema, M ${ }^{a}$ A. MARTíN, La estructura de la comunicación en Europa: las organizaciones internacionales, Madrid, Universidad Europea de Madrid/CEES. 
pres en Honduras y Agencia Nueva Nicaragua -ANN-en Nicaragua) e incluso un pool entre las mismas ${ }^{5}$. En esta línea, domina en cuanto a medios la agencia Prensa Latina (Cuba-1959) ${ }^{6}$.

La radiodifusión está ligada en su nacimiento a los capitales norteamericanos y europeos. Este hecho se reproduce con la aparición de la tecnología televisiva, destacando la presencia americana. Bien es cierto que su efecto fue positivo al permitir el desarrollo del medio en una fase temprana en Argentina, Brasil o México. Hay que constatar la existencia de matices en su grado de dependencia, así mientras que en Argentina las tres cadenas nacen unidas a las grandes emisoras americanas (CBS, ABC Y NBC), en Brasil su influjo se limita a una vinculación inicial con la Comisión Federal de Comunicaciones (EEUU). Con México la conexión es tecnológica como es el caso de Telesistema Mexicano (1955), aunque en la actualidad la NBC participe en la Televisión Azteca. Otros países latinoamericanos presenciaron el nacimiento televisivo unido al mundo universitario (Chile) o a la titularidad pública (Bolivia).

El sistema actual es de propiedad dual con la particularidad de que en Latinoamérica como en EEUU, primero se constituyen los canales privados y después los públicos, mientras que en Europa el fenómeno es inverso.

Una razón que explica lo que vamos diciendo, son las características técnicas de dichos medios. Las ondas se saltan con toda facilidad los marcos fronterizos. Así por ej., La Voz de América lanzada desde los Estados Unidos ha tenido gran influjo en todos los países Latinoamericanos. Fue un instrumento en manos de la CIA para oponerse a los movimientos comunistas propiciados y favorecidos desde la URSS. El comunismo hacía gran propaganda a través del servicio exterior de radio La Habana que llegó a ser el más importante de América Latina y el undécimo del mundo ${ }^{7}$. Al mismo tiempo, las estructuras organizativas de la radio y de la televisión comercial se han copiado de los Estados Unidos. Un tercer capítulo fue la ayuda al desarrollo. Desde los años cincuenta, numerosos gobiernos hispanoamericanos crearon y apoyaron diversos tipos de programas de desarrollo (extensión agraria, salud, educación...etc).

Hay que decir, con todo, que la radio, cuando opera entre fronteras, tiene efectos marginales. Esto se ha podido constatar en los casos en que ha actuado entre países cercanos. También cuando Moscú y Pekín lanzaban emisiones para América Latina. Los sondeos realizados demostraban que llegaban a un público

5 F. QUIRÓS, Curso de Estructura de la Información, Madrid, Dossat, 1991, p.171.

6 La agencia cuenta, según González Villanueva con: la publicación de las revistas de periodicidad mensual Prisma y Cuba Internacional, la difusión de boletines especializados como Panorama de la medicina o Directo sobre Cuba, una division de televisión (TV Latina), un servicio de radio y una agencia propia de publicidad (Publimagen). P. MARGOLLES, "Los medios y las condiciones en América Latina", en A.
FRAERMAN, Identidad y nuevos medios, Madrid, Comunica, 1995, p.199.

7 J. HALE, La radio como arma política, Barcelona, Gustavo Gili, 1979, p.131. 
muy limitado. La misma BBC de Londres ha tenido y sigue teniendo en América del Sur una audiencia muy escasa.

La transnacionalidad de la radio no se da en la prensa escrita cuya naturaleza es nacionalista, o como en el caso de Argentina y Brasil, capitalina. Toda ella está en manos de las oligarquías nacionalistas y presta atención a los fervores políticos que se dan en los distintos países. A pesar de ello se halla condicionada por los sistemas de información internacional. En Venezuela, por boca de Eleazar Díaz Rángel, se denuncia la dependencia de la información internacional y como «América Latina continúa siendo la región más ignorada en el resto del mundo... Igualmente están probadas la distorsión informativa, la omisión de hechos noticiosos importantes y la transmisión de noticias convenientes a los intereses del Departamento de Estado de EEUU, y en algunos casos, a los intereses de ciertos gobiernos ${ }^{8}$. A veces, son los poderes económicos y políticos, como ocurre en la de la misma Venezuela, los que presionan para que se mantengan las dependencias con respecto a los Estados Unidos.

\section{III)-. Proteccionismo cultural versus librecambismo}

En Europa se favorece el proteccionismo cultural por medio de los sistemas de cuotas de pantalla y de los Programas Media, dentro de la política audiovisual comunitaria y del canal Euronews (UER). Lo primero ocurre también en otras zonas, como Corea del Sur. La concepción latinoamericana -siguiendo la pauta de los Estados Unidos-, es más liberal. Ello, como es lógico, ha favorecido la americanización de las programaciones suscitando reacciones en contra.

En ese marco librecambista existen algunos medios y algunas programaciones que se han impuesto por sí mismos. Así, en el medio radiofónico, Cuba y Argentina fueron grandes exportadores de libretos de radio-teatro o radionovelas. En cuanto a la producción audiovisual, si bien no podemos hablar con propiedad de producción latinoamericana, al no existir una cinematografía con características regionales, sí que hay un género televisivo específicamente latinoamericano: la telenovela o el culebrón. Este género de ficción se realiza en América Latina desde el inicio de la actividad televisiva. De modo relevante en Argentina, Brasil, Colombia, Cuba, Chile, México, Puerto Rico, Perú y Venezuela. Esporádicamente en Ecuador y en Paraguay y en Los Angeles y Miami para el mercado hispano de Estados Unidos.

Su importancia es tal que ha provocado la traducción en áreas geográficas como Europa, Sudeste asiático, Estados Unidos u Oriente Medio. Pero esta transnacionalización ha supuesto, vía cooproducción o con la creación de sociedades especializadas, cambios en el género desde 1990. Lo que en su día fue visto como

8 E. DÍAZ RANGEL, La información internacional en América Latina, Caracas, Monte Avila Editores, 1991, p.241. 
medio de promoción del intercambio cultural y de la integración simbólica, se está convirtiendo en las últimas telenovelas en un fenómeno de hibridación o neutralización donde se pierden las marcas de identidad locales o regionales ${ }^{9}$.

\section{IV)-.Estatismo versus privatización.}

Tenemos que constatar aquí el fracaso en el desarrollo de políticas nacionales de comunicación. Los países en general, pero de manera especial los europeos, tenían su propia política de comunicación audiovisual creada por los gobiernos. El caso de la televisión privada británica era no sólo excepcional sino también limitado. La financiación de los medios se hacía por subvención, por canon o por publicidad.

El modelo norteamericano es completamente diferente pues es de dominio privado sobre la base de concesión de licencias hecha por la Comisión Federal de Comunicaciones (CFC).Dicho modelo se caracteriza por el escaso control estatal de los medios audiovisuales. Aunque existen emisoras públicas, éstas tienen una escasa cobertura.

Con el paso del tiempo, el modelo europeo ha ido evolucionando asemejándose más al norteamericano disminuyendo la presencia pública y aumentando la privada. Dicho proceso se conoce con el nombre de desreglamentación y fue provocado por la liberalización de las comunicaciones, el lanzamiento de los satélites y el desarrollo de los grupos de concentración.

Los países latinoamericanos siguieron el modelo estadounidense a la hora de ir estableciendo las grandes cadenas de comunicación audiovisual. Como dice Armand Mattelart, «mientras que Europa Occidental estaba todavía, en su totalidad, bajo el régimen del servicio y del monopolio público, los países latinoamericanos en su aplastante mayoría, vivían ya desde hacía años bajo el signo de la puja publicitaria, de las lógicas de la competencia e internacionalización de su programación $»^{10}$.

Sin embargo en los estudios por países se encuentran excepciones a dicha tendencia como es el caso del Perú en donde Canal 7 (Radio Televisión Peruana, RTP TV) es estatal. Otro caso singular es el de Nicaragua en donde la democracia mantuvo, durante el primer mandato, el estilo implantado por la revolución san-

9 Según N. MAZZIOTI, este fenómeno estaría definido por los siguientes rasgos: la desterritorialización, entendida como indefinición respecto al país de Hispanoamerica donde se realiza la serie, la pérdida de marcas lingüísticas identificatorias (por el idioma tampoco sabemos en que país transcurre la serie, la mezcla linguisticas identificatorias (por el idioma tampoco sabemos en que pais transcurre la serie, la mezcla en la utilización de acentos (argentino, venezolano, mexicano e hispano-neutro)y toda la telenovela aparece como un producto nociable cuando sus audiencias potenciales son ímprecisas y mudables, lo mismo orientadas al mercado latinoar cricano que a introducise en el nundo asiático, La industria de la telenovela pp. 26 y 113.

10 A. MATTELART, o.c., p. 203. 
dinista y tiene un sistema de propiedad mixto. Otros países como Venezuela tienen alguna cadena estatal y muchas otras privadas.

Caso aparte entre todos los demás es el de Cuba que sigue el modelo autoritario típico de la forma política socialista que, por excepción, permanece en aquel país. Esto nos lleva a hacer una mención sobre la libertad de prensa. La cuestión de la libertad tiene en América Latina una gran importancia pues hasta hace relativamente poco tiempo, en los años ochenta, se carecía, en muchos sitios de ella. En el caso del Brasil, la teoría que fundamentaba la dictadura allí establecida se llamó de la seguridad nacional. «La forma con que los generales de Brasilia han gestionado su relación con los medios ilustra bien a las claras, la tensión que, en los regímenes dictatoriales modernos, se introduce entre las normas de la guerra psicológica contra el enemigo interior -todo ciudadano es, de hecho, un sospechoso en potencia para el Estado de seguridad nacional- y las de la cultura comercial de masas que ambiciona e intenta seducir al público consumidor ${ }^{11}$.

\section{V)-. Civilización occidental versus otras civilizaciones.}

El sistema de desarrollo latinoamericano ha querido ser impulsado desde los Estados Unidos en diversas ocasiones a partir de patrones considerados como típicos de la civilización occidental. El ideal desarrollista de las décadas de los cincuenta y de los sesenta terminó en un fracaso lo que llevó a originar teorías en torno a la dependencia y dio base a la creación y difusión de la Teología de la Liberación que protestaba contra las grandes injusticias sociales. Las acciones revolucionarias hicieron que, como reacción, las dictaduras se implantaran en casi todos los países latinoamericanos. De esa forma la economía se estancó disparándose la deuda externa con lo que la década de los ochenta fue bautizada con el nombre de $<$ la década perdida $>$.

Esa doble concepción del desarrollo tiene para Huntington, como base, la realidad de una doble civilización: la occidental y la latinoamericana ${ }^{12}$ que quiso implantar sistemas corporativos (Juan Domingo Perón, en Argentina, Getulio Vargas en Brasil y el Méjico de la herencia cardenista), sobre la base de la concepción social de la escolástica española inspirada en los «cuerpos» de la Edad Media que ha conducido a facilitar el desarrollo del populismo y del caudillismo ${ }^{13}$.

Con la llegada de las nuevas democracias, la opción por el desarrollo se está realizando en Latinoamérica con métodos más aceptables y eficaces. Los medios de comunicación desempeñan un papel importante pues operan como agentes del desarrollo, modifican las conductas haciéndolas propias del tiempo actual y for-

11 A. MATTELART, o.c., p. 132.

12 S.P. HUNTINGTON, El choque de civilizaciones y la reconfiguración del orden mundial, Barcelona, Paidós, 1996, pp.51-52.

13 L.E. MANRIQUE, “América Latina y Occidente”, Politica Exterior, nº42, 1994/1995, p.176. 
man a las élites con la modernidad tecnológica. Por ello, al observar los cambios de la estructura comunicativa descubrimos su tendencia a la occidentalización como sucede con la economía (integración de México en la NAFTA, intensificación de las relaciones de Merco-Sur con la Unión Europea, celebración de las cumbres de las Américas y de las cumbres Iberoamericanas). Esa es la línea que Huntington desea y aconseja para el futuro: la integración paulatina de la civilización latinoamericana en la civilización occidenta ${ }^{14}$. Algunas de las empresas que detentan medios de comunicación desean alejarse de todo lo que tenga relación con el tercer mundo y acercarse a Occidente. Lo hallamos en Venezuela, Argentina, Colombia, etc.

\section{VI)-. Culturas propias versus cultura norteamericana.}

En el trasfondo de estas tendencias opuestas se halla la cuestión de la identidad. Los medios ejercen un influjo muy notable en el proceso de la formación y de la conservación de la identidad. Latinoamérica tiene unas peculiaridades propias fruto de una herencia española y portuguesa, mezclada con unas 39 etnias y 52 lenguas distintas cuyo conocimiento se quiere ahora potenciar con su presencia en la escuela y en los medios. Eso supone defenderse de los grandes productos industriales de comunicación importados de otra cultura. Así Mattelart y Durfan se preguntaban: «iPor qué Disney es una amenaza?. ... Porque este producto de Disneylandia ... es importado junto con tantos otros objetos de consumo al país dependiente. ....Importando un producto ... importamos también las formas culturales de esa sociedad» $»^{15}$.

Brasil y México tienen unas industrias muy poderosas de medios de comunicación. A pesar de ello, el desequilibrio entre productos exportados e importados es muy grande. México importa de los Estados Unidos el 30\% de su programación mientras que EEUU sólo importa el $2 \%{ }^{16}$.

En México los medios prestan particular atención a los numerosas, aunque poco extensas, culturas autóctonas. La Dirección General de Culturas Populares facilita estos servicios. Radio Comunal, emisora multilingüe es una buena muestra de tal empeño. Al producirse el levantamiento de Chiapas, se constató una división en los medios. Algunos no disimulaban su simpatía hacia los ideales propuestos por la población levantada.

\section{VII)-. Exposición ideológica directa versus métodos del entretenimiento.}

Todos los medios de comunicación son siempre ideología. Sin embargo, el método de transmitir contenidos ideológicos ha sufrido en estos últimos tiempos

14 S.P. HUNTINGTON, o.c., p. 374

15 A. DORFMAN y A. MATTELART, Para leer al Pato Donald, 1972, pp. 155-156

16 P. SCHLESINGER y N. MORRIS, "Comunicación e Identidad en América Latina", Telos, n49, p.56. 
una notable evolución. Por una parte, el debate ideológico tradicional se ha suavizado al tenerse que adaptar a las características de un medio como el televisivo donde «la información deja paso al espectáculo y por tanto, los acontecimientos sólo son noticiables si poseen un cierto impacto icónico» ${ }^{17}$.

La adaptación, sin embargo, no es el todo. A veces hay que hablar incluso de sustitución. Los programas ideológicos ceden muchísimo en audiencia a los dedicados al entretenimiento. Ricardo Vaca lo expresa así: «La <neotelevisión> es fusión de géneros televisivos. Los programas se presentan confundiendo, o mejor dicho mezclando las misiones hasta entonces bien diferenciadas; se informa divirtiendo, la información se convierte en <inforentretenimiento>, la publicidad es $<$ publireportaje $>$ o información de servicios y se toman prestadas fórmulas del espectáculo y del reclamo publicitario. Se eliminan los cortes tradicionales del programa, organizándose en torno a un flujo comunicativo que tiende a homogeneizar sus diferentes componentes $\rangle^{18}$.

Frecuentemente se ha constatado en Latinoamérica, la existencia de un desequilibrio entre oferta de los medios de comunicación y la demanda de sus públicos. Refiriéndose a la política de información, Angel Faus ha escrito que «no puede establecerse en función de planteamientos tecno-empresariales sino en función de las auténticas necesidades sociales hasta ahora ignoradas ${ }^{19}{ }^{\text {. }}$

Sobre el tema del entretenimiento se ha derramado ya mucha tinta lo que demuestra la valoración que de él se hace. Mikel Lejarza lo comenta así: «La ficción irá a más. A nosotros nos parece que la televisión es una máquina de contar historias, y como tal la ficción tendrá un sitio. Además la ficción tiene un elemento no sólo consustancial a esta filosofía sino algo más importante: la ficción es el elemento que puede dar personalidad propia a una cadena y además es el elemento que puede tener la cadena para salir de sus fronteras, para venderse ${ }^{20}$. El contraste entre los efectos de la tipografía en el «discurso maduro» y los efectos de la televisión como «desinformación», típico de la sociedad norteamericana como destaca Neil Postman, se dan también muy ampliamente en Latinoamérica ${ }^{21}$

Nos referimos aquí al contraste que aparece entre las necesidades y las expectativas de las audiencias y lo que los medios de comunicación ofrecen a las mismas. Esto se debe a varios factores. En primer lugar, la dificultad de dar a las masas lo que más les guste a lo largo de las veinticuatro horas del día durante todos los días del año. En segundo lugar, la fragmentación de la sociedad. Nunca los diferentes estilos de programas ofrecidos por las televisiones podrán gustar a

17 J.M. CONTRERAS, Vida política y televisión, Madrid, Espasa-Calpe, 1990, p.133.

18 R. VACA, Quien manda en el mando, Madrid. Visor, 1997, p.28.

19 La Era Audiovisual, Barcelona, Internacionales Universitarias, 1995, p.300.

20 R. VACA, O.C., P.416.

21 Divertirse hasta morir, Barcelona, Ediciones de la Tempestad, 1991, p.1991, p.300. 
todos. En tercer lugar, la diferencia de intereses. Las televisiones latinoamericanas están en manos de una oligarquía financiera e industrial que tiene intereses económicos y que defiende intereses políticos.

Tras hablar de los contrastes debemos recalcar sin embargo, el paralelismo que existe entre los medios impresos de los diversos países latinoamericanos. Jesús Timoteo Álvarez y Ascensión Martínez Riaza lo exponen con claridad: «En cada país han ido perdiendo peso los periódicos definidos como políticos (portavoces de un partido o de un grupo político), sustituidos por títulos que buscan prioritariamente el negocio. Ello no significa renunciar a una línea política propia, es más, hasta esa línea política suele ser común en los periódicos dominantes a que nos referimos: todos son moderadamente pro o antigubernamentales, todos moderadamente conservadores; todos son moderadamente conservadores en cuestiones económicas; todos son moderadamente progresistas en asuntos sociales» ${ }^{22}$.

\section{Identidad y Comunicación.}

Como ocurre en todo el mundo, actualmente, las comunidades humanas tienen sed de profundizar en su propia identidad. Para ello necesitan, primero, descubrirla y legitimarla; segundo hacer que la población la asimile como bien socio-cultural supremo. Esto es la causa de que las culturas indígenas estén siendo cada vez más protegidas a partir del reconocimiento de sus derechos. Estos movimientos no se han limitado a perspectivas puramente locales sino que en algunos casos han desarrollado planteamientos universales. Así por ejemplo, Brasil ha unido las demandas indígenas a la necesidad de proteger la selva amazónica. Las exigencias de los pueblos indígenas guatemaltecos adquirieron un alcance mundial con la concesión del premio Nobel a Rigoberta Menchú.

Esta aspiración en favor de la identidad se ve desarrollada a partir de poderosas acciones de carácter institucional:

$\left.1^{\circ}\right)$ La política de la UNESCO favorable a los medios de comunicación comunitarios. Los cuales valora especialmente.

Este tipo de medios comunitarios tienen ya una larga tradición y mantienen desde tiempo atrás un compromiso político como en el caso de Radio Mineras creada en 1947 en Bolivia. Son también medios de desarrollo educativo patrocinados a veces por fundaciones confesionales. Otras veces se utiliza el soporte radiofónico, el teatro popular o el foro del casete.

$\left.2^{\circ}\right)$ La prensa tiene un carácter marcadamente nacional, debido a la existencia de periódicos veteranos de élite como El Mercurio de Santiago de Chile, El Espectador de Bogotá, La Prensa de Buenos Aires y El Comercio de Lima.

22 Historia de la prensa hispanoamericana, Madrid, Mapfre, 1992, p.20. 
La formación de grupos de concentración multimediáticos, como ha sucedido en varios países pero de manera más relevante en Brasil, han sido un factor de identidad tanto para la formación de conciencia interior como para su identificación con el exterior.

$\left.3^{\circ}\right)$ La acción de la Iglesia latinoamericana especialmente a partir de la década de los setenta. Su interés en la adaptación, su acercamiento a las clases populares, su programación dirigida a la enseñanza, el fomento de movimientos y asociaciones dirigidos por ella ${ }^{23}$.

La construcción de la identidad latinoamericana ha sido notablemente impulsada desde que se inició la celebración de las cumbres iberoamericanas. En la Cumbre de Cartagena de Indias ( 8 de junio 1994), a través de un seminario taller dedicado a los medios de comunicación, se formularon las siguientes aspiraciones: 1). Intercambio de materiales periodísticos.2)-. Intercambio de iniciativas de producción y estudio. 3)-. Realización de análisis de la agenda informativa iberoamericana, por ejemplo, la recolección y distribución de los titulares de cada medio para su análisis, 4)-. la configuración y financiación de equipos de investigación. El objetivo es establecer la cooperación amplia y estable entre los medios iberoamericanos ${ }^{24}$.

Esa identidad tiene una manifestación en el acercamiento de las tecnologías y de los métodos. Uno de ellos es, obviamente, Internet. En el año 1994 América Latina alcanzó las 600 redes privadas con direcciones en Internet cifra que ha ido desde entonces en constante aumento. Se constató también la carencia de una Internet latinoamericana dado que los mensajes entre los distintos países siempre pasaban a través de Estados Unidos. Algunas investigaciones realizadas indican la existencia de limitaciones como la diferencia que se constata entre usuarios registrados y usuarios activos. Ello refleja el coste y la dificultad de utilizar redes en las naciones en vías de desarrollo.

\section{Integración, Cooperación y Comunicación.}

Latinoamérica, desde que se independizó de España y Portugal, a principios del siglo XIX, ha albergado numerosas propuestas en favor de su integración. Conocida es la acción de Simón Bolívar (Congreso Anfictiónico del Itsmo de Panamá, 1826). El integracionismo, hasta el momento, no ha tenido resultados positivos y el más alto nivel relacional que se ha conseguido ha sido de cooperación promovido por dos grandes movimientos: el panamericano y el interamericano. Una dualidad en la cual se encuentra inmerso el sistema latinoamericano de medios de comunicación.

23 E. FOX, Conclusiones, en E. FOX (ed), Medios de comunicación y politica en América Latina, Barcelona, 1989, pp.218.

24 A. FRAERMAN, "La búsqueda de las claves de identidad latinoamericana", en A. FRAERMAN (ed.), A. FRAERMAN, "La búsqueda de las claves de identidad
Identidad y nuevos medios, Madrid, Comunica, 1995, p.97. 
Según Miguel Rojas Mix, el panamericanismo es «un proyecto de integración sin identidad ${ }^{25}$. Fue impulsado por los Estados Unidos a partir de la Declaración de Monroe en 1823. Ha sido uno de los constantes objetivos de la política exterior norteamericana.

El interamericanismo parte de una concepción más igualitaria de los países latinoamericanos en relación con los Estados Unidos. En su marco se han concluido diversas realizaciones: Tratado Interamericano de Asistencia Recíproca (Río de Janeiro 1947), Tratado Americano de Soluciones Pacíficas (Bogotá, 1948) y la Organización de Estados Americanos (OEA).

Junto a esos esfuerzos de carácter global se han dado otros de carácter regional como el Pacto Andino, el Mercado Común Centroamericano y MercoSur. Una parte de Latinoamérica, en concreto México, ha dado preferencia a su cooperación con los países del Norte, dentro del Tratado de Libre Comercio de América del Norte (USA, Canadá, Méjico). Chile se encuentra en una situación ambivalente, sin haber decidido su adscripción ni al Tratado de Libre Comercio ni al Merco-Sur, los dos conjuntos regionales por los que se siente atraído.

En el campo de la comunicación los intentos de cooperación habidos hasta el momento pueden clasificarse de la siguiente forma:

1-. Intentos favorecidos por diversos organismos internacionales entre los que destaca la UNESCO. Como realizaciones concretas debemos destacar la Conferencia Americana Cult de Bogotá, finalizada el 30 de enero de 1978 y la Conferencia Intergubernamental de América Latina y el Caribe, celebrada en San José de Costa Rica en julio de 1976. Los principios allí valorados fueron los de identidad, desarrollo cultural y democratización.

2-. Realizaciones interconexas con sistemas exteriores a Iberoamérica. Así son, por ej., la Organización de la Televisión Iberoamericana (OTI), la Agencia Centroamericana de Notícias (ACAN-EFE) y la Caribbean News Agency (CANA). La OTI sigue las pautas técnicas de Eurovisión y la Acan está ligada a la Agencia EFE.

3-. Otros intentos lanzados por los propios países iberoamericanos como la Asociación de Sistemas Informativos Nacionales (ASIN), la Agencia SIP, el Sistema Económico Latinoamericano (SELA), la Agencia Latinoamericana de Servicios Especiales (ALASEI), la Unión Latinoamericana y del Caribe de Radiodifusión y la Asociación Interamericana de Radiodifusión (AIR).

4-. Existen, además, proyectos auspiciados por particulares como el de Gabriel García Márquez que fundó una escuela para periodistas latinoamericanos en Cartagena de Indias que en nuestros días sigue funcionando con éxito.

25 M. ROJAS, "Integración e identidad cultural", Integración y Comunicación, Madrid, Sociedad Estatal Quinto Centenario, p. 87. 
5-. Se desarrollan también acciones de carácter mixto como la de la Televisión Educativa Iberoamericana a la que contribuyen diversos canales de televisión, especialmente regionales, otras entidades como las universidades y los gobiernos de España y de países iberoamericanos. Su servicio se ofrece por Hispasat, en tres franjas de programación de dos horas de duración.

Por todo lo que hemos dicho hasta aquí, vemos que existe entre los medios de comunicación un verdadero esfuerzo de cooperación. La unidad lingüística de grandes áreas (castellano y portugués), y la gran cercanía que se da entre las dos lenguas facilita sobremanera la relación entre los medios. Hay que hablar aquí necesariamente del gran desarrollo que está teniendo lugar en la televisión vía satélite. Para América Latina 27 cadenas: Sur, Cartoon Network, Canal CNN International, Eco, Galavisión, Espil, International Fox, James Globosat, Hoptv, Tele 1, TNT, USAnews, Infinito, Júpiter, News music, Eritil, Aprima, Multishow, Telecine, NBC, Nuevoluce, Space, Telehits, Telenovelas, TopSpot, RAI y Televisión Española. Veinte de estas cadenas prestan servicio en lengua castellana, once en inglés, siete en portugués, diez pueden ser recibidas alternativamente en inglés o en castellano, una en italiano. Dichas cadenas utilizan quince satélites. España tiene en órbita -desde 1993- los satélites Hispasat IA e Hispasat IB. Desde el principio la Misión TV América fue prioritaria, asignándose a RTVE. Este ente ya tenía en funcionamiento el canal de TVE Internacional, que emitía para Europa y América parrillas diferenciadas. Esta última se completó con la creación del canal Hispavisión, en 1994. Con anterioridad, Brasil había lanzado el Brasilsat I, en 1985, que fue el primer satélite nacional de América Latina.

A pesar del esfuerzo al que antes nos referíamos no hay que olvidar que los medios siguen reflejando la realidad del capitalismo dependiente. Ello, sin embargo, no es un obstáculo a la cooperación sino que puede facilitar la misma. Lo que sí suele impedir, sin embargo, es una cohesión social más profunda.

\section{Iberoamérica y Sociedad multimedia interactiva.}

El concepto de sociedad multimedia se equipara al concepto de sociedad potsindustrial de Bell y de Touraine, al de sociedad tecnotrónica de Brizezinsky, al de sociedad de consumo de Jones y Baudrillard, al de sociedad informatizada de Nora Minc, al de sociedad interconectada de James Martin, al de estado telemático de Gubern, al de la tercera ola de Alvin Toffler, al de la aldea global de Mc Luhan y a los últimamente surgidos de sociedad digital de MerecierPlassard-Soardigly, Bustamante, Negroponte y Terceiro y de cibersociedad de Joyanes Aguilar ${ }^{26}$.

26 L. JOYANES, "Cibersociedad; la nueva sociedad de la información (Orígenes, evolución e implantación)", Sociedad y Utopía, no9, marzo 1997, p.84. 
Dicho mosaico de nombres supone la existencia de variedades en la realidad por ellos designada. Pero existen en ellos dos factores básicos que son comunes: la información y la innovación y un tercero, para nosotros consustancial: la participación del usuario-consumidor. Se suele entender también por multimedia la convergencia de la telefonía, la informática, el entretenimiento y la información $^{27}$.

Esta sociedad se caracteriza porque los medios otorgan el poder. Se pasa de «quien tiene el poder controla los medios» a «quien tiene los medios detenta el poder». Un poder que no es otorgado en las urnas, que está por encima de las fronteras y cuyo único freno es el deseo individual de cada usuario- consumidor.

Otra característica de dicha sociedad es que cuenta con una información universalizada que llega directamente al público, con el concurso secundario de las agencias de información. Es servida, bien a través de la cadena televisiva CNN o de la red de Internet, Ted Turner o la National Research and Education Network. Un medio privado y otro semipúblico garantizando ambos la política exterior y de seguridad norteamericana. Hasta ahora tenían en Latinoamérica un poder de uso limitado a las élites por la utilización del idioma inglés y por sus requisitos tecnológicos. La primera condición ya ha sido superada. Las cadenas de televisión CNN y CBS comenzaron a emitir a partir de 1996, programas informativos en español. «CNN en español» ofrece a Iberoamérica y España su nuevo canal de noticias en español, las 24 horas del día, contando con un corresponsal permanente en Cuba. «CBS Telenoticias» es una emisión en español dedicada a los 27 millones de latinos en Estados Unidos.

El abaratamiento de los costes de producción audiovisual a través del sistema digital es otro de los rasgos. Se trata de un sistema de potencialidad interactiva. Todo esto lleva consigo una transnacionalización de empresas y capital multimediático en la que los antiguos colonizadores resultan colonizados no sólo en su programación audiovisual sino en la concurrencia de la propiedad empresarial. En el caso de España, por Televisa y en el caso portugués por O'Globo que cuenta con una presencia del 15\% de capital en la Sociedad Independiente de Comunicación, propietaria de uno de los dos canales privados.

Las empresas españolas - Grupo 16, Grupo Prisa, Antena 3 Internacional y Recoletos han entrado ampliamente en Latinoamérica. Cambio 16 tuvo ediciones en México, Colombia y Chile. El grupo PRISA ha comprado varios diarios. En el caso de México, aunque vendió las acciones de Impulsora de Ediciones Periodísticas, editora del diario popular La Prensa, mantiene la sociedad editorial El País México que sigue publicando una edición facsimilar del periódico español matriz con un añadido de información local. El Grupo Recoletos adquirió en 1996 el 15\%

27 I. SANTILlana Del BARRIO, "Los Procesos de privatización. Presencia internacional de Telefónica", Telecomunicaciones 95. Tendencias, Madrid, Fundesco, 1995, p.193. 
de Ediciones Financieras propietaria del rotativo chileno de información económica El Diario. Antena 3 Internacional firmó un convenio, en 1996, finalmente no desarrollado con la empresa mexicana Multivisión para la creación de un nuevo canal, al 50\%, asumiendo la empresa española los contenidos y la mexicana la parte técnica. Antena 3 Internacional distribuiría la señal a través de DTH para toda Iberoamérica y a través de Telerey Multivisión se da la exclusividad de la señal para México, Centroamérica, Caribe, Venezuela, Colombia y Ecuador. En contrapartida, el Grupo Televisa inició desde 1996 una alianza estratégica con The News Corporation Limited, Organizacones Globo y Tele Communications International Inc. Opera no sólo en Latinoamérica sino que va dirigido al Caribe y al público hispano de U.S.A. y Canadá. Al final de 1996 dicha alianza inició en México las operaciones de DTH bajo el nombre de Sky Entertainnement Services. Su objetivo es transmitir a través de 150 canales su programación. Televisa está asociada en España con Telefónica, Radio Televisión Española, varias cadenas autonómicas y con grupos periodísticos. En Vía Digital tiene una participación del $25^{\prime} 45 \%$ (DTH Europa).

Por otra parte, Telefónica ha constituido con la Agencia EFE y con el grupo suizo Publícitas una empresa filial Hispaservices S.A. para el envío de publicidad. Viene así a ser esta empresa la primera integral de difusión publicitaria con destinatario multimedia que se constituye en todo el mundo.

La Red Globo de Televisión - la cuarta productora audiovisual del mundo, se ha asociado también con grandes grupos internacionales como HollywoodMGM, Paramount, Universal, Fox y USA Networks. De esa forma garantiza una programación vinculada a la cultura y a las realidades brasileñas y sirve de fomento a la producción nacional. 